\title{
Identifying Method for Key Quality Characteristics in Series-Parallel Mul- tistage Manufacturing Process
}

\author{
Wang Ning*
}

Business school, Zhengzhou University, No.100 of Science Road, Zhengzhou, Henan, Postcard: 450001, China

\begin{abstract}
Many actual multistage manufacturing processes are not serial structure, but parallel structure or hybrid structure. The quality relationship model for multistage manufacturing process with parallel structure was developed based on state space model. The quality relationship model of multistage manufacturing process with hybrid structure was developed via the hierarchical iteration method based on the models for the process with serial and parallel structures. The PLSR method was using for eliminating the correlation between quality characteristics to make analysis and identify the key quality characteristics. The method in the paper provides the solving basis for quality analysis and control in multistage manufacturing process.
\end{abstract}

Keywords: Hybrid Structure, Key Quality Characteristics, Multistage Manufacturing Process.

\section{INTRODUCTION}

The multistage manufacturing processes are common in modern production. The multistage process is defined as a system by which the product is made out through a series of process. Production is carried out by steps or stages. Each step in the manufacturing process directly or indirectly affects the whole process of quality assurance ability. There are a large number of quality characteristics in multistage manufacturing processes with a strong correlation between them. Due to cost and technological limitation, quality control personnel cannot monitor all quality characteristics. Therefore, we need to identify key quality characteristics to analyze and improve the quality of the multi-stage processes.

For key process quality characteristics identification of manufacturing, Boeing defined the key quality characteristics based on the degree of quality loss in the actual process of quality management (Boeing, 1991) [1-6]. The identification of key quality characteristics in multistage manufacturing process was developed in recent years. Ding (2005) uses the pattern recognition method to identify and monitor the quality variation in multistage production process of auto body. Tsung (2008) developed the identification method of key quality characteristics in the multistage process with state space wave transmission model. He and Tang (2007) developed the key quality characteristics based on house of quality method. Zhang et al. (2010) put forward the extraction model and optimization model of key quality characteristics for complex electromechanical product based on ANNs technology. Jin and Zhou (2006) developed a variation source identification method based on the analysis of the covariance matrix of the process quality measurements of manufacturing processes. Loose (2008) proposed the identification of key quality characteristics in multistage processes based on the eigenvectors of covariance matrix of multivariate measurements. But the researches focused on the single variable. The correlation between multiple quality characteristics was ignored (Wu, 2005) [7-15].

In addition, the present studies of identification key quality characters in multistage manufacturing process focuses only on the serial structure as the research object, not parallel structure. These cases are the most common form in the actual production [16-20].

\section{QUALITY RELATIONSHIP MODEL OF MULTI- STAGE MANUFACTURING PROCESS}

To establish the relationship of multistage manufacturing process quality model is the basis of the identification of key quality characteristics. There usually are serial and parallel structures in the multistage manufacturing process. The quality models of serial and parallel structures in multi-stage manufacturing process are respectively established in this paper. The two quality models were integrated to obtain the relationship of multistage manufacturing process with hybrid structure.

\subsection{Quality Relationship Model of Multistage Manufac- turing Process with Serial Structure}

The state space model is the most widely used to analyze the variation accumulation and transfer of quality characteristics in the multistage processes modeling (Thornton et al., 2000). The state space model was put forward by Jin and Shi (1999). Its core is the quality characteristics between various processes being described by the linear relationship in 2D vector set and superimposed with interference system, as shown in Fig. (1) [6,7]. 


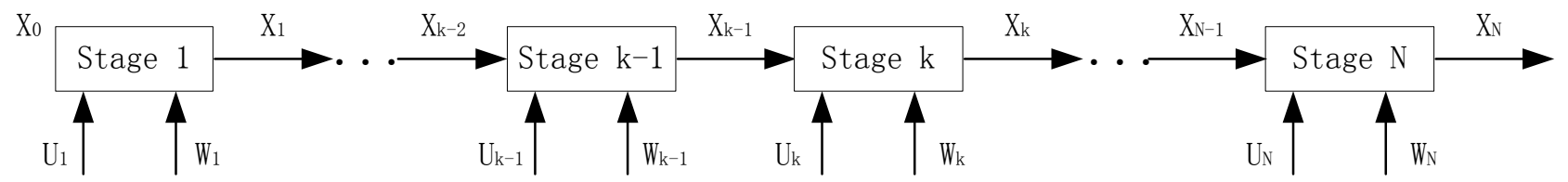

Fig. (1). Multistage process state space model.

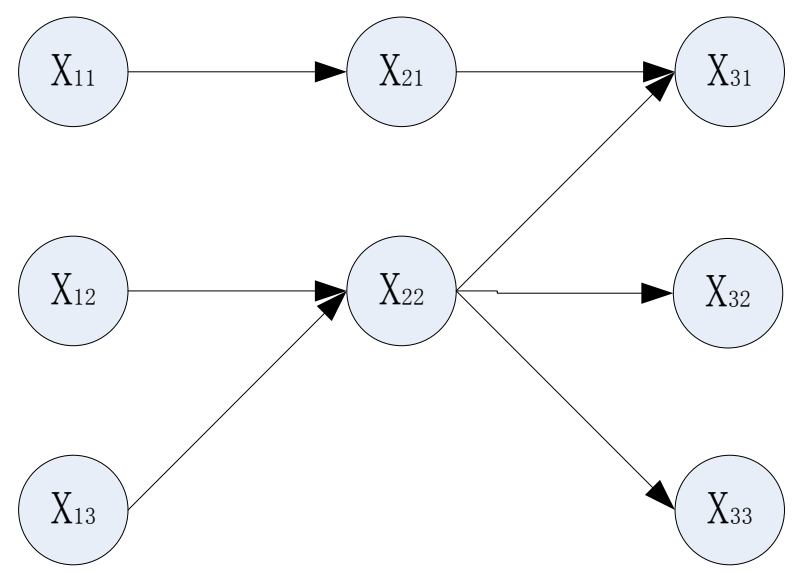

Fig. (2). The diagram of quality path in multi-stage manufacturing process.

Suppose a process has a total of $\mathrm{N}$ stages. The product quality characteristics vector $x_{k}$ of the stage k process consists of the quality characteristics vector $x_{k-1}$ and the quality influence $u_{k}$ from the stage. $x_{k-1}$ is not only the output of the stage k-1 quality characteristics, but also the input of quality characteristics in the stage $\mathrm{k} . u_{k}$ is independent. The random vector $w_{k}$ denotes random error variable, where the mean is zero. $w_{k}$ is independent to $u_{k}, k=1,2, \cdots, n$.

According to the linear state space model, quality measurement on the stage $\mathrm{k}$ of an in-control process is formulated by

$x_{i j}=\alpha_{i, j-1} x_{i, j-1}+\beta_{i j} u_{i j}+w_{j}$

Where, $x_{i j}(i=1,2, \cdots, m ; j=1,2, \cdots, k)$ denotes the ith quality characteristic of $j$ th stage. $\alpha_{i, j-1} x_{i, j-1}$ denotes the transformation of quality variation from the stage $j-1$ to the stage $j . \beta_{i j} u_{i j}$ denotes ith quality characteristics by the systemic factors in the stage $j$. The common-cause variation and random errors are represented by $w_{j}$ in the stage $j$ [21-23].

Quality relationship model of serial structure multistage manufacturing process can be formulated by vector as

$X_{j}=A_{j-1} X_{j-1}+B_{j} U_{j}+w_{j}$

Where, $X_{j}=\left(x_{1 j}, x_{2 j}, \cdots, x_{k j}\right)^{T}$ denotes $j$ th stage quality characteristic vector, $U_{j}=\left(u_{1 j}, u_{2 j}, \cdots, u_{m j}\right)^{T}$ denotes $j$ th stage quality characteristic influence. $A_{j-1}$ is influence coefficient matrix of quality characteristics from $j$-1th stage to the stage $j$, expressed as

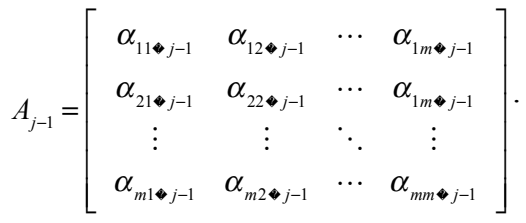

$B_{j}$ is influence coefficient matrix of quality characteristics from $j$ th stage, expressed as

$B_{j}=\left[\begin{array}{cccc}\beta_{11, j} & \beta_{12, j} & \cdots & \beta_{1 m, j} \\ \beta_{21, j} & \beta_{22, j} & \cdots & \beta_{2 m, j} \\ \vdots & \vdots & \ddots & \vdots \\ \beta_{m 1, j} & \beta_{m 2, j} & \cdots & \beta_{m m, j}\end{array}\right]$

\subsection{Quality Relationship Model of Multistage Manufac- turing Process with Parallel Structure}

In order to meet the need of productivity and production line balancing, multiple processes or station are often set up per stage, forming a parallel structure, in actual production. Fig. (2) is a reference of a typical parallel structure of multistage manufacturing process.

In the figure, the products from three stations will be the input of the two station of second phase. The products of two stations in second phase will be input of the third stage.

The state space model was studied only on the serial structure, not on parallel and hybrid structure of multi-stage 


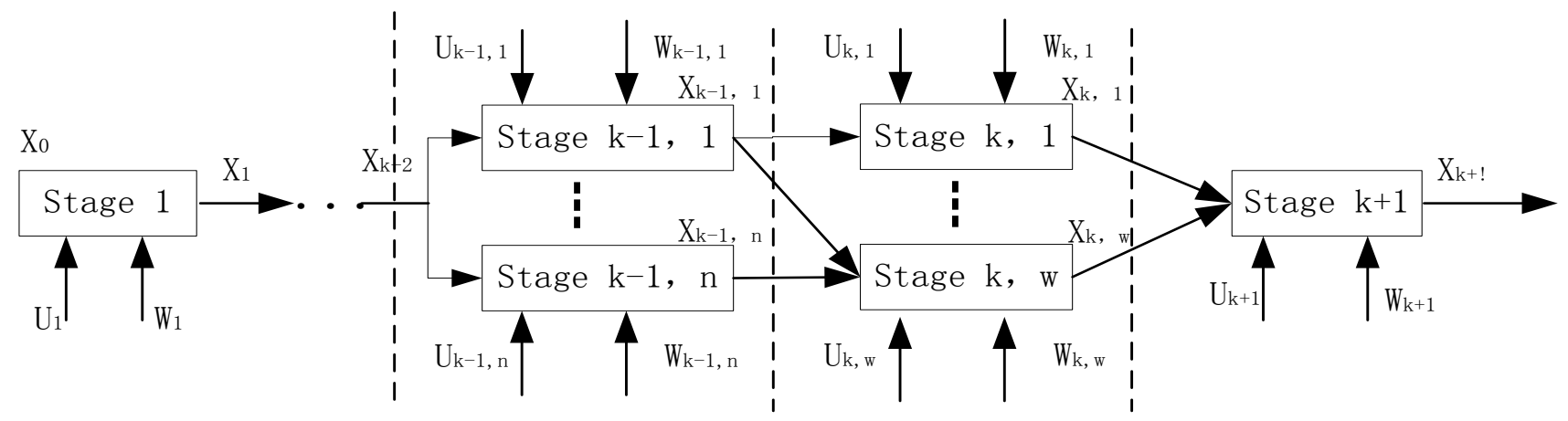

Fig. (3). The state space model of multi-stage manufacturing process with parallel structure.

processes. Jin, Li and Tsung (2010) researched the control method for parallel structure multistage manufacturing process. The traditional state space model is improved based on the idea of the paper to develop the key quality characteristic identification model for parallel structure multi-stage manufacturing process in the paper. Fig. (3) shows the state space model with parallel structure [24-26].

There are three case in the multi-stage manufacturing process with parallel structure as follow:

\section{a) The parallel case}

A parallel situation can be viewed as a cascade process which has two inputs without interaction. The quality relationship model still can be represented by state space model with serial structure. Through the recursive iteration, every parallel quality flow can be analyzed respectively to find out the influence on the process of quality characteristics of this process and setup linear relationship quality model of the entire manufacturing process.

b) The dispersion case

In the dispersion case, the output of the stage k-1 is the input of two stages in the stage $\mathrm{k}$. Two virtual variables $\tilde{X}_{k-1,1}$ and $\tilde{X}_{k-1,2}$ represents quality characteristics vector of the first station in the stage k-1 (Jin, 2010)[18],

$$
\begin{aligned}
& \left(\begin{array}{c}
X_{k, 1} \\
X_{k, 2}
\end{array}\right)=A_{k-1} X_{k-1}+U_{k}+w_{k}=\left(\begin{array}{cc}
A_{k-1} & 0 \\
0 & A_{k-1}
\end{array}\right) \\
& \left(\begin{array}{c}
\tilde{X}_{k-1,1} \\
\tilde{X}_{k-1,2}
\end{array}\right)+\left(\begin{array}{c}
U_{k, 1} \\
U_{k, 2}
\end{array}\right)+\left(\begin{array}{c}
w_{k, 1} \\
w_{k, 2}
\end{array}\right)
\end{aligned}
$$

Where, $\tilde{X}_{k-1,1}$ and $\tilde{X}_{k-1,2}$ represent virtual variable of $X_{k, 1}$ and $X_{k, 2}$ input. $\tilde{X}_{k-1,1}$ and $\tilde{X}_{k-1,2}$ has the same distribution. The variable $X_{k-1}$ is the superposition of $\tilde{X}_{k-1,1}$ and $\tilde{X}_{k-1,2}$ [27].

\section{c) The convergence case}

There are 2 inputs in the stage $\mathrm{k}+1$ from stage $\mathrm{k}$. Two virtual variables $\tilde{X}_{k+1,1}$ and $\tilde{X}_{k+1,2}$ represents quality characteristics vector of the first station in the stage $\mathrm{k}-1$.

$$
\begin{aligned}
& X_{k+1}=\left(\begin{array}{c}
\tilde{X}_{k+1,1} \\
\tilde{X}_{k+1,2}
\end{array}\right)=\left(\begin{array}{cc}
A_{k} & 0 \\
0 & A_{k}
\end{array}\right)\left(\begin{array}{c}
X_{k, 1} \\
X_{k, 2}
\end{array}\right) \\
& +\left(\begin{array}{c}
U_{k+1,1} \\
U_{k+1,2}
\end{array}\right)+\left(\begin{array}{l}
w_{k+1,1} \\
w_{k+1,2}
\end{array}\right)
\end{aligned}
$$

Where, $X_{k, 1}$ and $X_{k, 2}$ denote the quality characteristics of the stage k. The virtual variable $\tilde{X}_{k+1,1}$ and $\tilde{X}_{k+1,2}$ represents the influence from input $X_{k, 1}$ and $X_{k, 2}$, which has the same distribution. The variable $X_{k+1}$ is the superposition of $\tilde{X}_{k+1,1}$ and $\tilde{X}_{k+1,2}$. That is to say, the quality characteristics of the stage $\mathrm{k}+1$ are affected by the quality characteristics of the stage $\mathrm{k}$.

\subsection{Quality Relationship Model of Multistage Manufac- turing Process with Hybrid Structure}

Two adjacent stage quality characteristics can be represented by a linear state space model based on the above quality relationship. Therefore, the quality relationship model of multistage process with hybrid structure is developed through the hierarchical iteration method [28, 29].

$$
\begin{aligned}
& \left(\begin{array}{l}
X_{1,1} \\
X_{1,2} \\
X_{1,3}
\end{array}\right)=\left(\begin{array}{ccc}
A_{0} & 0 & 0 \\
0 & A_{0} & 0 \\
0 & 0 & A_{0}
\end{array}\right)\left(\begin{array}{l}
X_{0,1} \\
X_{0,2} \\
X_{0,3}
\end{array}\right)+\left(\begin{array}{c}
U_{1,1} \\
U_{1,2} \\
U_{1,3}
\end{array}\right)+\left(\begin{array}{c}
w_{1,1} \\
w_{1,2} \\
w_{1,3}
\end{array}\right) \\
& \left(\begin{array}{l}
X_{2,1} \\
X_{2,2}
\end{array}\right)=\left(\begin{array}{cc}
A_{1} & 0 \\
0 & A_{1}
\end{array}\right)\left(\begin{array}{c}
X_{1,1} \\
\tilde{X}_{1,2 \& 3}
\end{array}\right)+\left(\begin{array}{c}
U_{2,1} \\
U_{2,2}
\end{array}\right)+\left(\begin{array}{c}
w_{2,1} \\
w_{2,2}
\end{array}\right) \\
& \left(\begin{array}{l}
X_{3,1} \\
X_{3,2} \\
X_{3,3}
\end{array}\right)=\left(\begin{array}{ccc}
A_{2} & 0 & 0 \\
0 & A_{2} & 0 \\
0 & 0 & A_{2}
\end{array}\right)\left(\begin{array}{c}
\tilde{X}_{2,1 \& 2} \\
X_{2,2} \\
X_{2,2}
\end{array}\right)+\left(\begin{array}{l}
U_{3,1} \\
U_{3,2} \\
U_{3,3}
\end{array}\right)+\left(\begin{array}{c}
w_{3,1} \\
w_{3,2} \\
w_{3,3}
\end{array}\right)
\end{aligned}
$$

So, Y denotes the finial quality characteristic of the stage $\mathrm{k}$.

Based on formula (2), (3) and (4), the Y can be represented as 
$Y=A_{k-1} A_{k-2} \cdots A_{0} X_{0}+B_{k} U_{k}+A_{k-1} B_{k-1} U_{k-1}$

$+A_{k-1} A_{k-2} B_{k-2} U_{k-2}+\cdots+$

$A_{k-1} A_{k-2} \cdots A_{1} U_{1}+w_{k}+A_{k-1} w_{k-1}+A_{k-1} A_{k-2} w_{k-2}$

$+\cdots+A_{k-1} A_{k-2} \cdots A_{1} w_{1}$

Further, the formula can be expressed as

$Y=\sum_{i=1}^{k} \Phi_{k, i} B_{i} U_{i}+\Phi_{k, 0} X_{0}+\sum_{i=1}^{k} \Phi_{k, i} w_{i}$

Where, $\Phi_{(\cdot,)}$ is state transition matrix, while $k>i$, $\Phi_{k, i}=A_{k-1} A_{K-2}, \cdots, A_{i}$, while $k=i, \Phi_{k, k}=I \cdot X_{0}$ represents the original state before production. If the systemic random error $w_{j}$ is ignored and let $X_{0}=0$, the model (6) can be simplified as

$$
Y=\sum_{i=1}^{k} \Phi_{k, i} B_{i} U_{i}
$$

From the formula (8), the complex relation among quality characteristics of different stages can be expressed by state transfer relation. The quality of final product can be expressed by the quality characteristics of each stage of the process. The relationship among the $X_{j}, X_{j-1}$ and $U_{j}$ in a single stage need only be studied to setup the model. The quality relationship model of multi-stage manufacturing process with hybrid structure can be treated as linear equations of product quality $\mathrm{Y}$ and quality characteristics $U_{j}$ in each stage [30].

If the coefficient matrix effects can be obtained in each stage of quality characteristics, it can identify where the quality characteristics have more influence on the final product quality. That is the key quality characteristic of the process.

\section{KEY QUALITY CHARACTERISTIC IDENTIFYING AND MODELING ANALYSIS BASED ON PLSR}

The multivariate PLSR (Partial Least Squares Regression) method can be used to solve a number of related influence among the quality characteristics of multistage processes, to establish the regression model between the independent variable (input vector of quality characteristics of each stage) and dependent variable (the output vector of quality characteristics of each stage) (Wang, $\mathrm{Xu}$ and Yang, 2013)[8]. Through recursive iteration, the relationship model was setup between the quality characteristics of the final product and quality characteristics of each stage. Finally through the auxiliary technology in PLSR, the significant factors and the key quality characteristics can be identified. The identifying program is as follow:

\section{Step 1: Data collection}

Determine the final quality requirements and quality characteristics of multistage manufacturing process which has influence on the quality of the final product, and collect sample data. $\tilde{Y}$ is the independent variable and it represents the final quality of product. The dependent variable $\tilde{U}_{1}, \tilde{U}_{2}, \cdots, \tilde{U}_{K}$ denotes the quality characteristics of stage $k$. The data includes $n$ groups, that is $\left(\tilde{U}_{i 1}, \cdots, \tilde{U}_{i k}, \tilde{Y}\right), i=1, \cdots, n$.

\section{Step 2: Data standardization}

According to the formula (2), (3) and (4), the SIMCA-P software is used to do PLSR analysis in turn, from the beginning of the quality $\mathrm{Y}$ of the final product to input vector and output vector of each stage of processes with serialparallel structure. According to the aided analysis of PLSR, the information of variable importance can be obtained.

\section{Step 3: Quality relationship modeling}

According to the formula (5), (6) and (8), multiple linear regression model of product quality and the quality characteristics can be setup through recursive iteration from the quality characteristic $\mathrm{Y}$ of the final product to the upper stages of process. The regression model is the quality relationship model of multistage manufacturing process with hybrid structure. The model is expressed as

$\hat{Y}=\mu_{1} U_{1}+\mu_{2} U_{2}+\cdots+\mu_{k} U_{k}$

Where, $\mu_{i}(i=1, \cdots, k)$ is the Influence coefficient $\sum_{i=1}^{k} \Phi_{k, i} B_{i}$ of formula (8).

Step 4: The key quality characteristic identifying

The VIP (variable importance in projection) is the index to measure the importance of the dependent variable in PLSR. For each $U_{1}, \ldots, U_{k}$, the $U_{j}$ with $\operatorname{VIP}_{j}=1(j=1,2, \ldots, k)$ represents the importance of each $U_{j}$ for the quality characteristic $Y$ is the same. If the $U_{j}$ with $\operatorname{VIP}_{j}>1(j=1,2, \ldots, k)$, the $\mathrm{U}_{\mathrm{j}}$ can interpret the quality characteristic $\mathrm{Y}$ is better. The key quality characteristic can be identified through the value of VIP $_{\mathrm{j}}$. (Wang, 2006) [17].

\section{CONCLUSION}

The key quality characteristics identification method in this paper is developed for solving multistage manufacturing process with hybrid structure.

Firstly, the relationship of quality characteristic was discussed in the multistage manufacturing process. Three cases, that is parallel, dispersion and convergence case were discussed. Then the quality relationship model for multistage manufacturing process with parallel structure was developed based on state space model. The quality relationship model of multistage manufacturing process with hybrid structure was developed via the hierarchical iteration method based on the models for the process with serial and parallel structure. Because of the correlation between the quality characteristics, the identifying program is developed. The PLSR method was using for eliminating the correlation between quality characteristics. The VIP is defined as discriminated value to identify the key quality characteristics.

The method in the paper had been applied in the actual multistage manufacturing process in a cigarette firm. The 
results are proved to be effective. This method is still insufficient because it is based on lineal state space model. Mutual influence of quality characteristics is maybe nonlinear in multistage manufacturing process. It needs further study to solve the case.

\section{CONFLICT OF INTEREST}

The author confirms that this article content has no conflict of interest.

\section{ACKNOWLEDGEMENTS}

This paper was supported by the grants from Ministry of education of humanities and social science project (No.14YJC630126), Science and technology research key project in Henan province department of education (No. 14A630053) and the Humanities and social science research projects in Henan province department of education (2014GH-057).

\section{REFERENCES}

[1] Y. Ding, D. Ceglarek, and J. Shi "Fault diagnosis of multistage manufacturing processes by using state space approach", ASME Transactions, Journal of Manufacturing Science and Engineering, vol. 124, no. 2, pp. 313-322, 2002.

[2] Y. He, and X. Tang, "Design for quality based on product key quality characteristics", Acta Aeronautica et Astronautica Sinica, vol.11, no.6, pp.1468-1481, 2007.

[3] M.Jin, Y. Li, and F. Tsung, "Chart allocation strategy for serial-parallel multistage manufacturing processes", IIE Transactions, vol. 42, no. 8, pp. 577-588, 2010.

[4] N. Jin, and S. Zhou, "Data-driven variation source identification of manufacturing processes based on eigenspace comparison", Naval Research Logistics, vol. 53, no. 5, pp. 383-396, 2006.

[5] J-P Loose, S. Zhou, and D. Ceglarek, "Variation source identification in manufacturing processes based on relation measurements of key product characteristics", Journal of Manufacturing Science and Engineering, vol. 130, no. 3, pp. 1-11, 2008.

[6] J. Shi, and S. Zhou, "Quality control and improvement for multistage systems: a survey", IIE Transactions, vol. 41, pp. 744-753, 2009.

[7] A.C. Thornton, S. Donnelly, and B. Ertan, "More than just robust design: why product development organizations still contend with variation and its impact on quality", Research in Engineering Design, no. 12, pp. 127-143, 2000.

[8] N. Wang, J. Xu, and J. Yang, "Method of identifying key quality characteristics in multistage manufacturing process based on variation transmission model", Advances in Information Sciences and Service Sciences, vol.5, no. 2, pp. 208-214, 2013.

[9] X. Wang, "Method study of monitoring process disturbance based on integrated SPC/EPC", Advances in Information Sciences and Service Sciences, vol. 5, no. 5, pp. 345-352, 2013.

[10] F-C Wu, "Optimization of correlated multiple quality characteristics using desirability function", Quality Engineering, vol. 17, no.1, pp.119-126, 2005.

[11] L. Xiang, and F. Tsung, "Statistical monitoring of multistage processes based on engineering models", IIE Transactions, vol. 40, no. 10, pp. 957-970, 2008

[12] P.F. Zantek, G.P. Wright, and R.D. Plante, "Process and product improvement in manufacturing systems with correlated stages", Management Science, vol. 48, no. 5, pp. 591-606, 2002.
[13] G. Zhang, F. Ji, and X. Ren, "Key quality characteristics extraction model of complicated mechanical and electrical products", Journal of Chongqing University, vol. 2, no. 2, pp. 8-14, 2010.

[14] G. Zhang, "Multiple key quality characteristics optimization model for manufacturing process", Computer Integrated Manufacturing Systems, vol. 6, no. 16, pp. 1286-1291, 2010.

[15] S. Zhou, Y. Ding, Y. Chen, and J. Shi, "Diagnosability study of multi-station manufacturing processes based on linearmixed model", Technometrics, vol. 45, no. 4, pp. 312-325, 2003.

[16] S. Zhou, Q. Huang, and J. Shi "State space modeling of dimensional variation propagation in multistage machining process using differential motion vectors. IEEE Transactions on Robotics and Automation, 2003.

[17] H. Wang, Z. Wu, and J. Feng, "Linear and nonlinear partial leastsquares regression", National Defense Industry Press, China, 2006.

[18] S. Jing, W. Shengxian, and F. Zhihui, "Process capability analysis and estimation scheme for autocorrelated data", Journal of Systems Science and Systems Engineering, vol. 19, no. 1, pp. 105-127, 2010.

[19] W. Huang, and Z. Kong, "Process capability sensitivity analysis for design evaluation of multistage assembly processes", IEEE Transactions on Automation Science And Engineering, vol. 10, no. 7, pp. 736-745, 2010.

[20] F. Zhu, and X.F. Huang, "A complex product machining process capability performance improvement method", Applied Mechanics and Materials, vol. 11, pp. 652-655, 2011.

[21] D. Ceglarek, and J. Shi, "Fixture failure diagnosis for autobodyassembly using pattern recognition", ASME Transactions, Journal of Engineering for Industry, vol. 88, no. 1, pp. 55-65, 1996.

[22] Q. Rong, D. Ceglarek, and J. Shi, "Dimensional fault diagnosis for compliant beam structure assemblies", ASME Transactions, Journal of Manufacturing Science and Engineering, vol. 12, no. 4, pp. 773-780, 2000.

[23] M. Chang, and D.C. Gossard, "Computational method for diagnosis of variation-related assembly problem", International Journal of Production Research, vol. 36, no. 11, pp. 2985-2995, 1998.

[24] S. Zhou, Y. Chen, and J. Shi, "Root cause estimation and statistical testing for quality improvement of multistage manufacturing processes", IEEE Transactions on Automation Science and Engineering, vol. 1, no. 1, pp. 73-83, 2004.

[25] D. Ceglarek, and Shi, J, "Dimensional variation reduction for automotive body assembly", Journal of Manufacturing Review, vol. 8, no. 2 , pp. 139-154, 1995 .

[26] Z. Li, S. Zhou, and Y. Ding, "Patternmatching for root cause identification of manufacturing processes with consideration of general structured noise", IIE Transactions, vol. 39, no.3, pp. 251-263, 2007.

[27] Z. Li, and S. Zhou, "Robust method of multiple variation sources identification in manufacturing processes for quality improvement", ASME Transactions, Journal of Manufacturing Science and Engineering, vol. 128, no. 1, pp. 326-336, 2006.

[28] Z. Kong, D. Ceglarek, and W. Huang, "Multiple fault diagnosis method in multi-station assembly processes using orthogonal diagonalization analysis", ASME Transactions, Journal of Manufacturing Science and Engineering, vol.130, no. 1, pp. 128-130, 2008.

[29] Y. Ding, A. Gupta, and D. Apley, "Singularity of fixture fault diagnosis in multi-station assembly systems", ASME Transactions, Journal of Manufacturing Science and Engineering, vol. 126, no. 1, pp.200-210, 2004.

[30] M. Zhang, D. Djurdjanovic, and J. Ni, "Diagnosibility and sensitivity analysis for multi-station machining processes", International Journal of Machine Tools and Manufacture, vol. 47, no. 3, pp. 646-657, 2007. 